\title{
Leptina e sua influência na patofisiologia de distúrbios alimentares
}

\author{
Leptin and its influence in the pathophysiology \\ of eating disorders
}

Helen Hermana Miranda HERMSDORFF'

Maria Aparecida de Queiroga Milagres VIEIRA ${ }^{1}$

Josefina Bressan Resende MONTEIRO'

\section{R E S U M O}

A leptina é uma proteína secretada pelos adipócitos com papel regulador em vários sistemas do organismo, como sistema imune, respiratório e reprodutivo, bem como no balanço energético via ação hipotalâmica. Sua ação primária ocorre no núcleo hipotalâmico arqueado, no qual inicia uma cascata de eventos para inibição da ingestão energética e aumento do gasto energético. As concentrações de leptina são influenciadas pela adiposidade, fatores hormonais e nutricionais. A restrição e os episódios de compulsão alimentar, presentes na anorexia nervosa e bulimia, respectivamente, são considerados, na literatura científica, fatores determinantes na leptinemia. Seus níveis também alterados no tratamento desses distúrbios alimentares sugerem uma relação entre as alterações neuroendócrinas e conseqüentes modificações nos sinais de fome e saciedade, com a patogenia ou manutenção dos quadros clínicos. Trabalhos têm encontrado impacto dessas alterações na saúde dos pacientes, em curto e longo prazos. Esta revisão tem como objetivo esclarecer quais são as funções da leptina nos tecidos nervoso e periférico, quais os mecanismos que interferem na sua concentração nos distúrbios alimentares e como isso reflete na saúde do paciente anoréxico ou bulímico.

Termos de indexação: anorexia nervosa; bulimia; ingestão de alimentos; leptina.

A B S T R A C T

Leptin, a protein secreted by adipocytes, has a regulatory function in several organism systems such as the immune, respiratory, and reproductive systems and in the energy balance via hypothalamic action. Its primary action occurs in the arcuate hypothalamic nucleus, where it begins a cascade of events that inhibits energy intake and enhances energy consumption. The concentration of leptin is influenced by adiposity and hormonal and nutritional factors. The scientific literature considers food restriction and binge episodes of anorexia nervosa and bulimia, respectively, as determining factors of leptin circulation. Leptin levels are also altered during the treatment of these food disturbances, which indicates a relation between neuroendocrine alterations

\footnotetext{
1 Departamento de Nutrição e Saúde, Universidade Federal de Viçosa. Av. P.H. Holfs, s/n, Campus Universitário, 35671-000,

Viçosa, MG, Brasil. Correspondência para/Correspondence to: H.H.M. HERMSDORFF. E-mail: <helenhermana@yahoo.com.br>.
} 
and eventual modifications in hunger and satiety signals and pathogenesis or clinical state of patients. Studies have reported on the short and long-term impact of these alterations on health. Therefore, this review aims to explain the function of leptin in the central and peripheral nervous systems, the mechanisms that interfere with its concentrations in food disturbances, and how this reflects on the health of anorectic and bulimic patients.

Indexing terms: anorexia nervosa; bulimia; eating; leptin.

\section{N T R O D U Ç Ã O}

Em 1953, Kennedy ${ }^{1}$ propôs que um fator humoral, produzido por adipócitos, interferia negativamente na ingestão energética, em proporção ao grau de adiposidade corporal, agindo de forma direta no hipotálamo para modulação, por sua vez, do balanço de energia. Entretanto, as bases moleculares dessa hipótese lipostática não foram estabelecidas até o descobrimento, em 1994, do gene ob e da sua proteína codificada, denominada leptina, do grego leptos (magro)².

A leptina é um produto do gene ob que se encontra no cromossomo 7q31.3, no caso de humanos, e no cromossomo 6, em camundongos. O gene ob inclui $650 \mathrm{~kb}$ e está constituído por três exons separados por dois introns. A região que codifica a síntese de leptina se localiza nos exons 2 e 3, sendo esta uma proteína de 167 aminoácidos e com peptídeo terminal de 21 aminoácidos. Sua estrutura a classifica como da família das citocinas, tendo de 4 a 5 segmentos helicoidais ${ }^{3}$. A seqüência aminoacídica apresenta poucas diferenças interespécies, o que permite uma homologia entre a leptina humana de $84 \%$, com a proteína do camundongo, e de $83 \%$ com a leptina de ratos 4 .

A leptina tem sido implicada na regulação de vários sistemas, incluindo o sistema imune, respiratório, reprodutivo, hematopoiético e ósseo, além de seu papel no balanço energético, via sistema nervoso central $(\mathrm{SNC})^{5}$. Em relação ao balanço energético, tem como ação primária os neurônios no núcleo hipotalâmico arqueado (NHA), no qual estimula a expressão de neurotransmissores e hormônios ligados aos mecanismos de inibição da ingestão alimentar e aumento do gasto energético total, via ativação do sistema nervoso simpático. Ao mesmo tempo, inibe expressão do neuropeptídeo Y (NPY) e Agouti related peptide (AgRP), considerados orexigênicos, ou seja, envolvidos nos mecanismos de aumento da ingestão alimentar e de ação redutora no gasto energético ${ }^{6,7}$.

A anorexia nervosa (AN) e a bulimia (Bu), enfermidades caracterizadas por alterações nos padrões alimentares, apresentam, de forma relevante, distúrbios neuroendócrinos envolvendo o eixo hipotalâmico-hipofisário-gonadal ${ }^{8}$. Estudos têm tentado identificar e quantificar essas alterações, bem como avaliar as diferenças entre indivíduos anoréxicos, bulímicos e indivíduos sem história de distúrbios alimentares ${ }^{9,10}$. Outra linha de pesquisa em evidência é a de estudos comparando os pacientes em estado patológico e em tratamento, de forma a esclarecer como as alterações na leptina, e outros hormônios e neurotransmissores ligados à ingestão alimentar, podem interferir para a melhora ou manutenção do comportamento característico dos distúrbios alimentares ${ }^{3,11}$. Esses estudos são importantes, devido à necessidade de identificar o impacto na saúde e na qualidade de vida de indivíduos com distúrbios alimentares.

Esta revisão tem como objetivo, portanto, esclarecer quais são as funções da leptina nos tecidos nervoso e periférico, quais os mecanismos que interferem em sua concentração nos distúrbios alimentares e como isso reflete na saúde dos pacientes anoréxicos ou bulímicos.

LEP T IN A

\section{Fatores determinantes dos níveis séricos}

Os níveis séricos da leptina são influenciados pela adiposidade, por fatores nutricionais 
e hormonais ${ }^{12}$. A adiposidade tem sido relatada como principal fator determinante da leptinemia, correlacionada em estudos com a massa total de gordura e o índice de massa corporal (IMC) ${ }^{6}$. Em relação aos diferentes depósitos de gordura corporal, o tecido adiposo subcutâneo tem maior produção e secreção de leptina, o que pode ser comprovado pelas determinações de leptina nos adipócitos de diferentes depósitos in vitro e in vivo $^{12,13}$, enquanto que a gordura intra-abdominal apresentou melhor associação com a presença de menor sensibilidade insulínica ${ }^{14}$. Em relação à ingestão alimentar, menores concentrações de leptina são obtidas após horas de restrição alimentar ou jejum de 12 horas, enquanto que 1 hora após a ingestão alimentar, as concentrações de leptina estão elevadas ${ }^{10}$.

Concomitantemente à ingestão alimentar, as alterações na insulinemia parecem alterar a leptinemia, sendo este hormônio pancreático, um dos reguladores hormonais da secreção de leptina pelo tecido adiposo ${ }^{13}$. Em ratos, infusões de insulina aumentam as concentrações de leptina, enquanto que em ratos $d b / d b$, com quadro de resistência insulínica, as concentrações são reduzidas ${ }^{15}$. Em culturas de adipócitos, a ação da insulina por si só não altera a secreção da leptina e, sim, seu efeito sobre o metabolismo da glicose nos mesmos, especialmente no transporte de glicose. A metabolização da glicose nos adipócitos envolve glicólise, lipogênese e conversão da mesma em glicosamina, via D-frutose-6-fosfato-aminotransferase, sendo esses mecanismos estimuladores da produção de leptina (transcrição do gene, expressão do ácido ribonucléico (RNA) mensageiro e secreção da proteína leptina) ${ }^{4}$. Dessa forma, os efeitos da restrição ou realimentação, de reduzirem ou aumentarem as concentrações da leptina, respectivamente, de forma independente da gordura corporal, podem estar relacionadas às alterações no metabolismo de glicose no tecido adiposo mediado pela insulina. O consumo de pré-carga de carboidrato ou lipídio tem efeitos diferentes sobre a leptinemia, sendo que a ingestão de lipídio resulta em menor secreção de leptina, o que aumenta a ingestão energética e o risco de obesidade ${ }^{4,15}$. Estudos têm comprovado a existência de receptores de leptina no pâncreas, com efeito inibitório sobre a liberação de insulina, via ativação de canais de potássio sensíveis à adenosina trifosfato (ATP) ${ }^{3,16}$. Sugere-se, por esses resultados, a existência de um mecanismo de feedback entre pâncreas e tecido adiposo.

O cortisol, aumentado em restrições alimentares, inibe a secreção diurna da leptina, via redução da sensibilidade insulínica, e o efeito da insulina no metabolismo de glicose no tecido adiposo $^{14}$. Tais efeitos são coerentes com o ciclo circadiano da leptina, com maiores concentrações por volta da uma hora e menores em torno de nove horas da manhã ${ }^{16}$.

Outros hormônios podem alterar os níveis séricos e cerebroespinhais da leptina, como os estrógenos, estimuladores, e catecolaminas e andrógenos (testosterona), inibidores da secreção da leptina ${ }^{3,17}$. Citocinas, a saber, interleucina 1 (IL-1), interleucina 6 (IL-6) e fator tumoral $\alpha$ (TNF- $\alpha$ ), também interferem na expressão do RNA mensageiro, mas os mecanismos não estão bem esclarecidos ${ }^{8,18}$.

Desse modo, a composição corporal, a composição da dieta e outros fatores endócrinos e metabólicos, envolvidos no balanço energético, interferem na leptinemia em estados de jejum e pós-prandiais. A presença de concentrações alteradas de leptina, em indivíduos em estado de $\mathrm{AN}$ e $\mathrm{Bu}$, está diretamente relacionada às alterações ocorridas nesses fatores.

\section{Ação da leptina no sistema nervoso central e tecidos periféricos}

A leptina tem sido implicada na regulação dos sistemas imune, respiratório, reprodutivo, entre outros tecidos e sistemas, e no balanço energético via SNC 5 .

a) Sistema Imune: Receptores para leptina estão expressos nas células hematopoiéticas nas 
quais a leptina produzida pelas células adiposas marrom, estimula o desenvolvimento normal de mielóides e eritróides ${ }^{3}$. Além disso, a leptina age sinergicamente com outras citocinas melhorando a proliferação de leucócitos, especificamente das células T4.

b) Sistema Respiratório: A leptina, em ratos, age como fator de crescimento no pulmão e como modulador nos mecanismos de controle da respiração central. Níveis de leptina são elevados em pacientes com apnéia, independentemente da gordura corporal, sendo associados à resistência leptínica ${ }^{3}$.

Os estudos ainda não são conclusivos a respeito dessas áreas de atuação da leptina, mas, baseados nos autores citados neste trabalho, pode-se mostrar que a ação dessa proteína é muito mais complexa e envolve mais tecidos que o proposto quando descoberta.

c) Sistema Reprodutivo: Em ratos ob/ob, a administração de leptina leva ao aumento da secreção de gonadotrofinas com retorno da fertilidade, indicando a ação dessa proteína na função gonadal e na fertilidade ${ }^{4,16}$. A leptina poderia agir no hipotálamo para estimulação da produção de hormônios gonadotróficos.

Na puberdade, o aumento da secreção de leptina é diretamente proporcional ao aumento da gordura corporal, principalmente no sexo feminino. A leptina age tanto no hipotálamo como na glândula hipófise para secreção dos hormônios luteinizante $(\mathrm{LH})$ e folículo estimulante (FSH), sugerindo seu envolvimento na menarca como sinal ou mensagem ao hipotálamo de que a reserva de gordura é suficiente para uma gestação ${ }^{16}$. A hipótese de que a leptina modula a atividade gonadal é ainda suportada pela melhora significativa em anormalidades menstruais, níveis séricos de estrógeno e testosterona, e resposta do LH ao hormônio liberador de LH, em mulheres com deficiência congênita de leptina, também caracterizada por lipodistrofia e anormalidades no sistema reprodutivo nessas pacientes ${ }^{19}$.

As concentrações de leptina são diferentes entre os sexos, sendo superiores para as mulheres, a partir da puberdade, e de forma permanente após maturação sexual. As explicações para esse dimorfismo sexual são a porcentagem de gordura corporal superior nas mulheres e a ação inibitória do hormônio testosterona na secreção de leptina no sexo masculino ${ }^{16,20}$. Rump et al..$^{21}$ apresentaram, em seu estudo, um aumento da concentração de leptina nas gestantes durante o parto, chegando, inclusive, a se duplicar, e uma redução deste hormônio após o parto, concomitante a um processo de dessensibilização dos receptores hipotalâmicos, o que vem sendo relacionado à dificuldade para perda de peso no pós-parto.

d) Balanço Energético: A leptina, juntamente com a insulina, é considerada um modulador lipostático que regula a ingestão e o gasto energético a longo prazo em relação ao estoque de gordura corporal ${ }^{18,22}$.

Os receptores da leptina, denominados receptores ob-r, são incluídos na família das citocinas e possuem várias formas identificadas, mas uma, em especial, encontra-se em grande quantidade no hipotálamo, especificamente no NHA (ob-Rb), o que o indica como local de importante ação da leptina ${ }^{3}$. Esses receptores têm três partes funcionais: (1) Extracelular, que interage com a proteína leptina e que é idêntica a todas as isoformas dos receptores ob-r; (2) Intracelular, que, quando ativada, estimula os eventos celulares. Seu tamanho e seu domínio determina a ação que a leptina exercerá sobre a célulaalvo ${ }^{23}$. Após ativação, mediada pelo sistema chave-fechadura com a proteína leptina, age sobre Janus quinase (Janus kinase -JAK), que fosforila proteínas membros da família de transcrição e tradução de sinais (Signal Tranduction And Transcription - STAT). Essas proteínas agem dentro do núcleo das células, ativando transcrição de genes dependentes de leptina, mecanismo denominado efeito genômico da leptina; (3) Transmembrana, que serve para "ancoramento" do receptor à membrana celular 7,23 .

A ação primária da leptina ocorre no NHA, em duas populações de neurônios distintas: (1) Neurônios que expressam pró-opiomelanocortina 
(POMC), precursor do hormônio estimulante dos melanócitos ( $\alpha$-MSH); (2) Neurônios que expressam a cocaína e a anfetamina, pela regulação da enzima transcriptase (CART). Todas as substâncias expressas nesse sistema são anorexigênicas, ou seja, agem na redução da ingestão alimentar ${ }^{3,6}$. A leptina estimula a produção dessas substâncias para balanço energético negativo. (2) Neurônios que expressam o NPY e o AgRP, relacionados ao aumento da ingestão alimentar e da eficiência metabólica para menor gasto energético ${ }^{6}$. O NPY é um peptídeo de 36 aminoácidos, sintetizado principalmente no NHA, e que se projeta para os núcleos hipotalâmicos para ventricular (NHP), ventromedial (NHVM), perifornical (NHF) e lateral $(\mathrm{NHL})$, também envolvidos na regulação do balanço energético. É o mais potente orexigênico. O AgRP também é sintetizado no NHA e projetado para os NHP, NHVM e NHL. Ele age como análogo no sistema melanocortina nos receptores MC-3 e MC-4, estimulando a ingestão alimentar. A leptina age sobre os neurônios do NHA, inibindo a expressão e biossíntese desses neuropeptídeos ${ }^{24}$.

Entre as ações promovidas pelos outros núcleos hipotalâmicos em relação à homeostase energética, pode-se destacar o NHVM como centro da saciedade, NHL como centro da fome e NHP ligado a efeitos para aumento do gasto energético, como produção de hormônios liberador de corticotrofina $(\mathrm{CRH})$ e liberador de tirotrofina (TRH), ativadores do sistema nervoso simpático. No NHF, há produção de peptídeos denominados orexinas $A$ e $B$, que agem no NHVM, inibindo a saciedade e aumentando a ingestão. Essas áreas recebem axônios de núcleos de neurônios do NHA, POMC/CART e NPY/AgRP, sendo considerados áreas de ação secundária da leptina (downstream) $)^{3,23}$. A função da leptina como reguladora no SNC tem, portanto, mecanismo desencadeante (sentido cascata), de modo a estimular ou inibir substâncias capazes de agir direta ou indiretamente nas áreas hipotalâmicas envolvidas no controle do balanço energético que, por sua vez, agem para tal controle.

Outra ação da leptina no balanço da ingestão energética é sua integração com o peptídeo sinalizador da saciedade, a curto prazo. A colescitoquinina (CCK) é liberada no intestino, após ingestão de uma refeição, e age no nervo vagal aferente para ativar neurônios no NHVM e reduzir o tamanho da refeição. A leptina agiria em neurônios no núcleo tractus solitarus, potencializando a ação da CCK para acelerar o término da refeição ${ }^{14,22,25}$. O peptídeo YY (PYY), sintetizado nas células endócrinas $L$ do intestino em resposta ao conteúdo calórico ingerido, é capaz, como a leptina, de inibir os neurônios NPY/ AgRP e estimular os neurônios POMC/CART no $\mathrm{NHA}$, com conseqüente efeito de redução na ingestão alimentar ${ }^{26}$. A leptina poderia, portanto, ter também efeito sinérgico a essa outra proteína intestinal. A longo prazo, parece também modular o limiar da fome suprimindo a ghrelina, um peptídeo gástrico com ação orexigênica. A reciprocidade da redução dos níveis séricos da leptina e do aumento de ghrelina, com a redução da adiposidade, nos leva à hipótese de que a leptina reduz os efeitos orexígenos, via ação no SNC e redução da ghrelina, via ação periférica ${ }^{27}$. Dessa forma, a leptina teria uma conexão direta com neurônios no trato gastrointestinal para a resposta de sinais relacionados à ingestão energética.

Em relação ao gasto energético, estudos têm encontrado associação positiva entre leptina e ativação do SNS em ratos, primatas e humanos ${ }^{5}$. A administração de leptina no terceiro ventrículo aumenta a produção de norepinefrina, neurotransmissor simpático ${ }^{4}$. Além disso, a leptina estimula a oxidação de ácidos graxos e captação de glicose em tecidos não adiposos, prevenindo a lipotoxicidade causada pelo acúmulo excessivo de triglicerídios em tecidos, como músculo, fígado, coração e pâncreas. Ela age inibindo a atividade da carboxilase acetil-coenzima A e ativando da carnitina palmitol transferase 1 , via ação no eixo hipotalâmico-simpático ${ }^{28}$.

Os estudos em modelos animais e humanos indicam, portanto, o papel da leptina no balanço energético, seja pela inibição da ingestão seja pelo aumento do gasto energético, importante para manutenção do peso corporal e prevenção 
da obesidade. Ainda apresentam ações desse hormônio em tecidos, órgãos e sistemas de todo o organismo para maior funcionalidade dos mesmos, o que suporta a hipótese de que a leptina tem ação central (hipotálamo) e periférica.

\section{Alterações séricas da leptina e seu impacto nos distúrbios alimentares}

A anorexia nervosa ( $A N$ ) pode ser definida como uma desordem alimentar caracterizada pela redução na ingestão energética, aumento da atividade física, perda de peso crônica e resistência ao tratamento para ganho de peso ${ }^{29}$. Em acréscimo, a bulimia (Bu) caracteriza-se por episódios de ingestão excessiva de alimentos, períodos prolongados de jejum e outros comportamentos compensatórios (grande freqüência de vômitos provocados e uso de laxantes e diuréticos) para reduzir a ingestão ou aumentar o gasto energético ${ }^{30}$. A Bu se diferencia da AN por atingir grupo de indivíduos com maior faixa etária e com maior prevalência de sobrepeso e obesidade ${ }^{31}$. Apesar de diferentes alterações comportamentais, ambas são distúrbios alimentares, em que a preocupação com o peso é intensa e a imagem corporal, distorcida ${ }^{32}$. Pesquisas têm sido realizadas com o intuito de identificar as alterações no eixo hipotalâmico-hipofisário-gonadal, presentes nas duas enfermidades. No que se refere ao eixo hipotalâmico, os estudos tentam relacionar as alterações dos sinais de apetite e saciedade no sangue e fluido cerebroespinhal e seus efeitos no desenvolvimento e, ou manutenção dos distúrbios alimentares 8,9 .

As concentrações séricas e no fluido cerebroespinhal de leptina, em pacientes anoréxicos, são geralmente reduzidas em relação aos indivíduos incluídos no grupo controle ${ }^{32,33}$. Isso se deve, primariamente, à redução de massa gorda, refletida também na redução do IMC, em pacientes com restrição alimentar. Segundo Tolle et al. ${ }^{34}$, mulheres constitucionalmente magras apresentaram níveis intermediários de leptina, quando comparados aos de anoréxicas e do grupo controle. Tal resultado coincidiu com uma porcentagem de gordura também intermediária nas mesmas, ou seja, menor que a do grupo controle e maior que a das anoréxicas, que, além de redução grave da gordura corporal, apresentaram alterações em hormônios sexuais. Licinio et al. ${ }^{35}$ mostraram que as mulheres necessitam de uma concentração de leptina duas vezes mais alta que os homens, sendo as primeiras relativamente mais resistentes aos efeitos da leptina. Isso pode explicar o aumento da susceptibilidade aos distúrbios, tanto alimentares, como do peso corporal.

Em ratos, uma restrição de $50 \%$ das necessidades energéticas levou à redução de $20 \%$ do peso corporal e $75 \%$ das concentrações de leptina ${ }^{10}$. Em estudo com humanos, mulheres anoréxicas apresentaram concentrações plasmáticas de leptina $70 \%$ menores do que as de mulheres do grupo controle $(1,9 \pm 1,5 \mu \mathrm{g} / \mathrm{l} \mathrm{e}$ $7,8 \pm 3,0 \mu \mathrm{g} / \mathrm{l}$, respectivamente). Nesse mesmo estudo, as concentrações de leptina dos dois grupos foram ajustadas para porcentagem de gordura corporal, por meio de análise estatística de covariância. Os valores obtidos após correção foram menores (em $\mu \mathrm{g} / \mathrm{l})$ em ambos os grupos, ou seja, se somente o tamanho de tecido adiposo interferisse na magnitude da leptinemia, teríamos valores menores na leptinemia para a porcentagem de gordura encontrada nas mulheres anoréxicas desse estudo ${ }^{36}$. A determinação da leptinemia em anoréxicas recuperadas, ou seja, em estado nutricional estabilizado, foi significantemente menor que em mulheres que nunca apresentaram algum distúrbio alimentar. Esses valores foram associados, não à composição corporal e sim a uma menor ingestão energética e a aspectos psicológicos, como direção à magreza, perfeccionismo, insatisfação corporal ${ }^{37}$. Além disso, o aumento dos níveis de leptina após tratamento pode ocorrer de forma mais tardia ao ganho de peso e à recuperação do IMC normal ${ }^{38}$. O resultado desses estudos sugere a presença de outros fatores determinantes na secreção de leptina em pacientes com AN, além do estoque de gordura, como fatores dietéticos e psicológicos. 
A restrição do macronutriente carboidrato, especificamente, poderia diminuir a oferta de glicose para o metabolismo nos adipócitos e, conseqüentemente, reduzir a produção de leptina ${ }^{15}$. As citocinas, como TNF- $\alpha$ e IL-1, também podem agir diretamente no hipotálamo, ou indiretamente, por meio de neurotransmissores monaminérgicos (serotonina) e na secreção de leptina ${ }^{8,18}$. A intensidade e a gravidade do quadro clínico, bem como níveis aumentados de cortisol, podem contribuir para redução da secreção da leptina ${ }^{39}$. A ghrelina, peptídeo produzido no estômago, também pode exercer ações metabólicas opostas à leptina. Ela estimula efeitos orexigênicos e adipogênicos, via modulação de NPY/AgRP, enquanto a leptina os inibe. Em condições de balanço energético negativo, como na anorexia nervosa, a ghrelina seria positivamente regulada (up regulation), em contraste à regulação negativa da leptina (down regulation), de modo a promover um mecanismo adaptativo para equilíbrio do balanço energético ${ }^{34}$.

Os pacientes em tratamento apresentam aumento nos níveis séricos da leptina, geralmente associados ao ganho de peso e gordura corporal, sendo sugerido, por alguns autores, como indicador do estado nutricional de pacientes anoréxicos ${ }^{3}$. Em outros estudos, as áreas da curva da leptinemia, em resposta ao tratamento de realimentação e ganho de tecido adiposo, tendem a atingir a normalidade precocemente, ou ainda a serem maiores que as do grupo controle, contribuindo para o quadro de AN e dificultando a sustentação do peso norma|8,11,32,40. Mulheres que apresentaram quadro de anorexia acompanhado por 10 anos após tratamento, tinham porcentagem de gordura corporal significantemente menor que mulheres que nunca tiveram algum distúrbio alimentar ${ }^{41}$. Essa diferença na composição corporal pode ter ocorrido pela presença de comportamento ainda alterado, como maior freqüência e intensidade na atividade física e restrição alimentar, mesmo que não em estágio patológico ${ }^{37}$, além da hipótese de que a restrição energética na adolescência tenha limitado a capacidade de acúmulo de gordura na fase adulta ${ }^{41}$.
Na bulimia, os níveis de leptina são maiores que na $A N$, mas reduzidos em relação ao grupo controle. Em comparação às bulímicas, as obesas apresentam valores ainda superiores ${ }^{42}$. Estudos têm mostrado uma tendência para desinibição e suscetibilidade para fome mais proeminente em bulímicas obesas, seguidas das não obesas, podendo a menor concentração de leptina ou a resistência à mesma, presente em obesos, estarem relacionadas com a patogenia e, ou manutenção do comportamento alimentar alterado na $\mathrm{Bu}^{42,43}$. A inversa correlação entre leptina sérica e freqüência de episódios de compulsão alimentar é coerente com a hipótese de que a leptina interfere no comportamento bulímico. Em tratamento, os níveis da leptina estão abaixo do grupo controle, podendo interferir no controle desses episódios ${ }^{11}$. Outros autores afirmam, ainda, que a freqüência de episódios de compulsão e vômitos e a quantidade de comida ingerida podem interferir na secreção de leptina ${ }^{30,43}$. Esses resultados sugerem que baixos níveis de leptina levam a episódios bulímicos, tendo os mesmos, juntamente com a quantidade de alimentos ingeridos, ação estimulante na secreção de leptina, mas não de forma suficiente e, ou eficiente para atingir um comportamento alimentar normal.

Além disso, outros mecanismos podem interferir no comportamento alimentar na AN e Bu. Alterações de $\beta$-endorfina, gastrina, somatostatina e CCK também são relatadas na anorexia ${ }^{44}$. Valores de CCK, por exemplo, estão aumentados e reduzidos na AN e Bu, respectivamente, sugerindo sua influência na redução da ingestão e no processo de realimentação na AN e no aumento de hiperfagia nos episódios de compulsão na Buํ. Essas evidências podem contribuir para a compreensão dos profissionais envolvidos no tratamento desses pacientes a respeito da dificuldade de adesão ao mesmo, tendo não somente a interferência psicológica característica do quadro de distúrbio alimentar, mas também alterações que, provenientes dos próprios distúrbios, dificultam o retorno ao comportamento alimentar normal. 
O neuropeptídeo $Y$, produzido no hipotálamo e com efeito regulador na ingestão alimentar, também é estudado nos distúrbios alimentares. Banks et al. ${ }^{6}$ encontraram níveis séricos de NPY elevados na bulimia, podendo também estar relacionados a hiperfagia. Segundo hipótese apresentada por Inui ${ }^{9}$, na anorexia nervosa o NPY, orexinas e AgRP estariam aumentados como efeito compensatório, de modo a aumentar a ingestão e o ganho de peso. Mas suas ações estariam comprometidas pelo excesso de componentes anorexigênicos, como CRH, CCK e TNF- $\alpha$, indicando um desequilíbrio no circuito hipotalâmico regulador da ingestão energética.

A serotonina, também envolvida na modulação do comportamento alimentar, tem sua atividade avaliada pela determinação do nível cerebroespinhal de seu principal metabólito, ácido 5-hidroixindolacético $(5-\mathrm{HT})^{45}$. Em pacientes com AN, a concentração de 5-HT apresenta-se reduzida, enquanto que em pacientes em tratamento apresenta-se acima da média dos indivíduos controle $^{3,45}$. A redução da serotonina poderia estar relacionada com a redução na ingestão de aminoácidos essenciais, conseqüentemente do triptofano, precursor da serotonina, bem como na sensibilidade do receptor serotoninérgico (5HT-2C), envolvida na regulação feita pela IL-1 e leptina ${ }^{18,46}$. O polimorfismo de genes associados à produção e secreção de serotonina tem sido estudado, podendo a ação alterada da serotonina nos distúrbios alimentares ter possível etiologia genética ${ }^{31}$.

A leptina, na AN e Bu, apresenta, portanto, alterações nos seus níveis séricos e cerebroespinhais de forma concomitante a alterações de outros hormônios e neuropeptídeos, bem como da composição corporal, do comportamento alimentar e do perfil psicológico. Os resultados dos estudos sugerem que essas alterações agem de forma sinérgica e direcionada a menor ingestão alimentar e menor ganho de peso em pacientes em recuperação, aumentando a chance de reincidências e de comprometimento das funções fisiológicas. Mais estudos são necessários para explicar como os aspectos envolvidos podem interferir na leptinemia e, por sua vez, na ação desse hormônio na AN e Bu. Além disso, não está esclarecido se alterações dos neuropeptídeos, hormônios e citocinas, presentes nessas enfermidades, têm efeito sinérgico ou antagônico à leptina na modulação do balanço energético.

O impacto da redução da leptina não está somente ligado ao seu papel regulador na ingestão alimentar, como descrito anteriormente. Estudos têm encontrado correlação positiva na redução da leptina com redução nos hormônios sexuais ${ }^{16,39,42}$. Goumenou et al. ${ }^{18}$ encontraram relação positiva entre redução da leptina com secreção de LH em pacientes anoréxicas em tratamento, sugerindo que a leptina possui mecanismo envolvido na secreção de gonadotróficos.

A amenorréia, sintoma clínico comum entre mulheres com AN e Bu, também pode estar relacionada com menores concentrações da leptina. A redução de pelo menos 15\% do tecido adiposo e leptinemia abaixo de $3 \mathrm{ng} / \mathrm{ml}$ podem causar distúrbios menstruais ${ }^{16}$. Mesmo após a recuperação de peso e ingestão alimentar, as anoréxicas tratadas parecem apresentar função do eixo hipotalâmico-hipofisário-gonadal comprometida, caracterizada por ausência de padrão secretório e menores concentrações de LH e menores concentrações plasmáticas de estradiol. Essas alterações parecem estar ligadas a níveis séricos de leptina ainda reduzidos ${ }^{37}$.

Em homens, a redução na leptinemia leva a baixos níveis de testosterona e menores desejos e desempenho sexual, tendo melhoras na secreção de testosterona e LH com aumento da leptina. Apesar dos andrógenos terem efeito negativo na secreção da leptina, nos pacientes anoréxicos, não foi encontrado esse efeito, estando a leptina mais relacionada a outros mecanismos de expressão e produção ${ }^{20}$.

Alterações no sistema imune, também comuns nos distúrbios alimentares, são refletidas pela redução de linfócitos e citocinas. Esses resultados são coerentes com a alta prevalência de infecções nesses indivíduos ${ }^{47}$. 
Por último, a redução de leptina pode estar ligada a redução no gasto energético. A leptina estimula o gasto energético por meio da ativação do SNS, expressão de proteínas mitocondriais responsáveis por oxidação de substrato e supressão do NPY. Para isso, são necessárias concentrações bem acima das encontradas nos distúrbios alimentares, diminuindo o gasto energético total na $\mathrm{AN}$ e Bu ${ }^{10,36}$. Associada a esses mecanismos, a perda significativa de tecido muscular, processo mais proeminente na $\mathrm{AN}$, indica também redução do gasto energético ${ }^{36}$.

\section{CONSIDERAÇÕ ES FINAIS}

Esta revisão pôde identificar alguns fatores que têm influência sobre os níveis séricos da leptina, de forma integrada, podendo destacar a adiposidade, a ação da insulina e o metabolismo de glicose nos adipócitos, bem como ação de outros hormônios (cortisol, estrógenos e andrógenos) e das citocinas (IL-1, IL-6 e TNF- $\alpha$ ). A ingestão alimentar também tem papel determinante na leptinemia, tanto em comportamentos alimentares normais como nos distúrbios estudados, AN e Bu.

Os níveis séricos da leptina apresentam-se alterados nessas enfermidades, podendo estar envolvidos nos mecanismos de manutenção da restrição ou compulsão alimentar, ou ainda, na dificuldade de adesão dos pacientes ao tratamento. Além do papel regulador na ingestão energética, tem sido discutida a ação da leptina nos sistemas respiratório, imune e reprodutivo, sendo que as alterações séricas da leptina, em pacientes com $\mathrm{AN}$ e $\mathrm{Bu}$, podem comprometer a função desses sistemas de forma crônica, com piora na qualidade de vida dos mesmos.

É importante destacar que a ação da leptina nos tecidos nervoso e periférico não ocorre isoladamente no organismo, devendo-se considerar, portanto, a influência de outros fatores no poder de ação da leptina, na ingestão alimentar e nas alterações metabólicas.
Mais trabalhos científicos são necessários para o esclarecimento das alterações séricas da leptina em estados de jejum e pós-prandiais. Os efeitos da ingestão de macronutrientes e do conteúdo energético da dieta, associados aos outros hormônios envolvidos no balanço energético, bem como à adiposidade e outras características (idade, sexo) dos participantes dos estudos são aspectos relevantes para o entendimento dos mecanismos envolvidos na leptinemia, e devem, portanto, ser bem controlados para a obtenção de evidências significativas. Os fatores determinantes da leptinemia são alterados em indivíduos com distúrbios alimentares, o que torna necessários os estudos em indivíduos acometidos por essas enfermidades, para avaliar o quanto as mesmas interferem indiretamente na leptinemia e o quanto essas alterações nos níveis de leptina podem prejudicar a adesão ao tratamento e a qualidade de vida dos pacientes anoréxicos e bulímicos.

\section{REFER Ê NCIAS}

1. Kennedy GC. The role of fat depot in the hypothalamic control of food intake in the rat. Proc Roy Soc London Biol Sci.1953; 140(901): 578-96.

2. Zhang YY, Proenca R, Maffei M, Barone M, Leopold $\mathrm{L}$, Friedman JM. Positional of the mouse obese gene and its human homolog. Nature. 1994; 372(6505):425-32.

3. Janeckova R. The role of leptin in human physiology and pathophysiology. Physiol Res. 2001; 50(5):443-59.

4. Havel PJ. Peripheral signals conveying metabolic information to the brain: short-term and long-term regulation of food intake and energy homeostasis. Exp Biol Med. 2001; 226(11):963-77.

5. Havel PJ. Mechanisms regulating leptin production: implications for control of energy balance. Am J Clin Nutr. 1999; 70(3):305-6.

6. Banks WA, Lebel CR. Strategies for the delivery of leptin to the CNS. J Drug Target. 2002; 10(4): 297-308.

7. Niswender KD, Schwartz MW. Insulin and leptin revisited: adiposity signals with overlapping physiology and intracellular signaling capabilities. Front Neuroendocrinol. 2003; 24(1):1-10. 
8. Eckert E, Pomeroy C, Raymond N, Kohler PF, Thuras $P$, Bowers CY. Leptin in anorexia nervosa. J Clin Endocrinol Metab. 1998; 83(3):791-5.

9. Inui A. Eating behavior in anorexia nervosa- an excess of both orexigenic an anorexigenic signaling? Mol Psychiatry. 2001; 6(6):620-4.

10. Velkoska E, Morris MJ, Burns P, Weisinger RS. Leptin reduces food intake but does not alter weight regain following food deprivation in the rat. Int J Obes. 2003; 27(1):48-54.

11. Frederich R, HU S, Raymound N, Pomeroy C. Leptin in anorexia and bulimia nervosa: importance assay technique an method of interpretation. J Lab Clin Med. 2002; 139(2):72-9.

12. Cnop M, Landchild MJ, Vidal J, Havel PJ, Knowels NG, Carr DR, et al. The concurrent accumulation of intra-abdominal and subcutaneous fat explains the association between insulin resistance and plasma leptin concentrations. Diabetes. 2002; 51(4):1005-15.

13. Park KG, Park KS, Kim MJ, Kim HS, Suh YS, Ahn JD, et al. Relationship between serum adiponectin and leptin concentrations and body fat distribution. Diabetes Res Clin Pract. 2004; 63(2):135-42.

14. Blevins JE, Schwartz MW, Baskin DG. Peptide signals regulating food intake and energy homeostasis. Can J Physiol Pharmacol. 2002; 80(5):396-406.

15. Havel PJ. Role of adipose tissue in body weight regulation: mechanisms regulating leptin production and energy balance. Proc Nutr Soc. 2000; 59(3):359-71.

16. Goumenou AG, Matalliotakis IM, Koumantakis GE, Panidis DK. The role of leptin in fertility. Eur J Obstetr Ginecol Reprod Biol. 2003; 106(2):118-24.

17. Havel PJ, Kasim-Karakas GRD, Mueller W, Johnson PR, Gingerich RL, Stern JS. Relationship of plasma leptin to plasma insulin and adiposity in normal weight and overweight women: effects of dietary fat content and sustained weight loss. J Clin Endocrinol Metab. 1996; 81(12):4406-13.

18. Sato T, Laviano A, Meguid MM, Chen C, RossiFanelli F, Hatakeyama K. Involvement of plasma leptin, insulin and free trytophan in cytokineinduced anorexia. Clin Nutr. 2003; 22(2):139-46.

19. Oral EA, Ruiz E, Andewelt A, Sebring N, Wagner AJ, Depaoli AM, et al. Effect of leptin replacement on pituitary hormone regulation in patients with severe lipodystrophy. J Clin Endocrinol Metab. 2002; 87(7):3110-7.

20. Wabitsch M, Ballauff A, Holl R, Blum WF, Heinze E, Remschmidt $H$, et al. Serum leptin, gonadotropin, and testosterone concentrations in male patients with anorexia nervosa during weight gain. J Clin Endocrinol Metab. 2001; 86(7):2982-8.
21. Rump P, Otto SJ, Hornstra G. Leptin and phospholipid-esterified docosahexaenoic acid concentrations in plasma of women: observations during pregnancy and lactation. Eur J Clin Nutr. 2001; 55(4):244-51.

22. Baskin DG, Figlewicz LD, Seeley RJ, Woods SC, Porte D, Schwartz MW. Insulin and leptin: dual adiposity signals to the brain for the regulation of food intake and body weight. Brain Res. 1999; 848(1-2): 114-23.

23. Schwartz MW, Woods SC, Porte DJR, Seeley RJ, Baskin DG. Central nervous system control of food intake. Nature. 2000; 404(6778):661-71.

24. Morton GJ, Schwartz MW. The NPY/ArRP neuron and energy homeostasis. Int J Obes. 2001; 25(Suppl 5):S56-62.

25. Peters JH, Karpiel AB, Ritter RC, Simasko SM. Cooperative activation of cultured vagal afferent neurons by leptin and cholecystokinin. Endocrinology. 2004; 145(8):3652-7.

26. Tovar SA, Seoane LM, Caminos JE, Nogueiras R, Casanueva FF, Diéguez C. Regulation of peptide YY levels by age, hormonal, and nutritional status. Obes Res. 2004; 12(12):1944-50.

27. Ueno N, Dube MG, Inui A, Kalra PS, Kalra SP. Leptin modulates orexigenic effects of ghrelin and attenuates adiponectin and insulin levels and selectively the dark-phase feeding as revelealed by central leptin gene therapy. Endocrinology. 2004; 145(9):4176-84.

28. Gil-Campos MG, Canete R, Gil A. Hormones regulating lipid metabolism and plasma lipids in chilhood obesity. Int J Obes. 2004; 28(Suppl 3): $575-80$.

29. Feurté S, Nicolaidis S, Gerozissis K. Is the early increase in leptinemia one of the anorectic signals induced by essential amino acid-deficient diet in the rat? Endocrinology. 2000; 141(10):3916-9.

30. Monteleone P, Bortolotti F, Frabrazzo M, La Rocca A, Fuschino A, Maj M. Plasma leptin response to acute fasting and refeeding in untreated women with bulimia nervosa. J Clin Endocrinol Metab. 2000; 85(7):2499-503.

31. Fairburn CG, Harisson PJ. Eating disorders. Lancet. 2003; 361(9355):407-16.

32. Gendall KA, Kaye WH, Altemus M, McConaha CW, La Via MC. Leptin, neuropeptide $Y$, and peptide YY in long-term recovered eating disorder patients. Biol Psychiatry. 1999; 46(2):292-9.

33. Krivoza J, Papezova H, Haluzikova D, Parizkova J, Jiskra J, Kotrli Haas T, et al. Soluble leptin receptor levels in patients with anorexia nervosa. Endocrinol Res. 2002; 28(3):199-205. 
34. Tolle $V$, Kaden M, Bluet-Pajot MT, Jure D, Foulon C, Bossu C, et al. Balance in ghrelin and leptin plasma levels in anorexia nervosa patients and constitutionally thin women. J Clin Endocrinol Metab. 2003; 88(1):109-16.

35. Licinio J, Negrão AB, Mantzoros C. Sex differences in circulating human leptin pulse amplitude: clinical implications. J Clin Endocrinol Metab. 1998; 83(11):4140-7.

36. Polito A, Jabbri A, Ferro-Luzzi A, Cuzzolaro M, Censi L, Ciarapica D, et al. Basal metabolic rate in anorexia nervosa: relation to body composition and leptin concentrations. Am J Clin Nutr. 2000; 71(6): 1495-502.

37. Brambilla F, Monteleone P, Bortolotti F, Dalle Grave $R$, Todisco $P$, Javaro A, et al. Persistent amenorrhoea in weight-recovered anorexics: psychological and biological aspects. Psychiatry Res. 2003; 118(3):249-57.

38. Shimizu T, Satoh $Y$, Kaneko $N$, Susuki M, Lu T, Tanaka $\mathrm{K}$, et al. factors involved in the regulation of plasma leptin levels in children and adolescents with anorexia nervosa. Pediatr Int. 2005; 47(2): 154-8.

39. Nakai Y, Hamagaki S, Kato S, Seino Y, Takagi R, Kurimoto F. Leptin in women with eating disorders. Metabolism. 1999; 48(2):217-20.

40. Holtkamp K, Hebebrand J, Mika C, Heer M, Heussen $\mathrm{N}$, Herpertz-Dahlmann B. High serum leptin levels subsequent to weight gain predict renewed weight loss in patients with anorexia nervosa. Psychoneuroendocrionology. 2004; 29(6):791-7.

41. Frey J, Hebebrand J, Muller B, Ziegler A, Blum WF, Remschmidt $H$, et al. Reduced body fat in long-term followed-up female patients with anorexia nervosa. J Psychiatric Res. 2000; 34(1): 83-8.

42. Monteleone P, Frabrazzo M, Tortorella A, Fuschino A, Maj M. Opposite modifications in circulating leptin and soluble leptin receptor across the eating disorder spectrum. Mol Psychiatry. 2002; 7(6): 641-6.

43. Adami GF, Campostano A, Cella F, Scopinaro N. Serum leptin concentration in obese patients with binge eating disorder. Int J Obes. 2002; 26(8):1125-8.

44. Baranowka B, Radzikowska M, WasilewskaDziubinska E, Roguski K, Borowiec M. Disturbed release of gastrointestinal peptides in anorexia nervosa and in obesity. Diabetes Obes Metab. 2000; 2(2):99-103.

45. Ramacciotti CE, Coli E, Paoli R, Marazziti D, Dell'Osso L. Serotonergic activity measured by platelet [3H]paroxetine binding in patients with eating disorders. Psychiatry Res. 2003; 118(1): 33-8.

46. Kaye WH, Barbarich NC, Putnam K, Gendall KA, Fernstrom J, Fernstrom M, et al. Anxiolytic effects of acute tryptophan depletion in anorexia nervosa. Int J Eat Disord. 2003; 33(3):257-67.

47. Nova E, Samartin S, Gomez S, Morande G, Marcos $A$. The adaptive response of the immune system to the particular malnutrition of eating disorders. Eur J Clin Nutr. 2002; 56(Suppl 3):S34-7.

Recebido em: 18/5/2004

Versão final reapresentada em: 4/7/2005 Aprovado em: 28/7/2005 